\title{
PARTICIPACIÓN CIUDADANA EN LA GOBERNANZA DE LOS ESPACIOS NATURALES PROTEGIDOS DE ANDALUCÍA: EL CASO DEL PARQUE NATURAL BAHÍA DE CÁDIZ
}

\author{
J.M. Barragán Muñoz y J.A. Chica Ruiz \\ Facultad de Ciencias del Mar y Ambientales \\ Av. República Saharaui, s/n, 11.510 Puerto Real \\ Cádiz, Universidad de Cádiz \\ juan.barragan@uca.es \\ adolfo.chica@uca.es
}

\begin{abstract}
Resumen: Si bien en la actualidad el paradigma de la gestión de los espacios protegidos tiende a un modelo en el que los actores sociales sean capaces de intervenir en la toma de decisiones de manera activa, existen pocos estudios empíricos que corroboren tales tendencias. Es por ello que en este trabajo se estudia la participación pública en un espacio concreto: el Parque Natural Bahía de Cádiz (PNBC). Para su desarrollo se utiliza metodología cuantitativa y cualitativa, a partir de la realización de un cuestionario, elaborado exclusivamente para los miembros de la Junta Rectora del espacio protegido, y el análisis de diferentes documentos públicos de origen institucional (actas de las Juntas Rectoras, normativa existente, memorias, etc.). La investigación permite hacer un examen crítico tanto de algunas cuestiones que afectan a la gobernanza interna del espacio protegido como del marco general de referencia en el que se inscribe este caso particular (la Comunidad Autónoma de Andalucía), y que puede ser igualmente relevante en el ámbito nacional. Las recomendaciones finales apuntan a buscar mejoras en los estándares de gobernanza de nuestro capital natural.
\end{abstract}

Palabras clave: gobernanza, participación, Andalucía, Parque Natural de la Bahía de Cádiz.

Abstract: Although nowadays the protected areas paradigm tends to a model in which citizens are able to participate actively in decision-making process, there are few empirical studies which corroborate such trends.

Recibido: 20-7-2015. Aceptado: 20-10-2015. 
That is why in this paper public participation is analyzed in a concrete area: the Bay of Cadiz Natural Park. To do so, quantitative and qualitative methodology is applied, based on the exploitation of a questionnaire exclusively designed for the Board members of this protected area, along with the scrutiny of different institutional public documents (meeting minutes, regulations, reports, etc.). This research allows a critical examination of different aspects affecting the internal governance of the protected area, as well as of the general framework in which this particular case occurs (Andalucia), which can be equally relevant within the national ambit. Final recommendations are aimed to seek improvement in standards for the governance of our natural capital.

Keywords: governance, participation, Andalucia, Bay of Cadiz Nature Park.

\section{Introducción}

La conveniencia de un mayor protagonismo de la participación ciudadana en la gestión de los espacios protegidos, como elemento clave para su gobernanza, concita cierto acuerdo entre investigadores e instituciones internacionales (Abrams et al., 2003; Dearden, Bennett y Johnston, 2005; Pomeroy, Parks y Watson, 2006; Secretaría de la Convención de Ramsar, 2010; Jones, Qiu, y De Santo, 2011; Borrini-Feyerabend et al., 2013). También sucede algo parecido para el caso de los espacios protegidos de España y Andalucía (Europarc-España, 2002, 2007, 2014; García y Montes, 2010). El paradigma predominante ha ido evolucionando a lo largo de las últimas décadas. Si mediada la anterior centuria la protección de especies era la razón de ser de determinadas políticas públicas, la conservación de biotopos o la gestión integrada, sustituyeron de forma progresiva los fundamentos de actuación. Hoy día el paradigma tiende a estar más cerca de un modelo en el que los actores sociales sean capaces de intervenir, de manera más clara, en el sistema de toma de decisiones (Becken y Job, 2014).

Con tales antecedentes generales se aborda el principal objetivo de este trabajo: perfilar la participación pública en la gestión del Parque Natural Bahía de Cádiz (PNBC). Dicho perfil se interpreta como una aproximación a lo que podría estar sucediendo en los espacios protegidos de Andalucía de esta categoría. El órgano colegiado específico para facilitar la participación ciudadana es la Junta Rectora (JR). Por esta razón el esfuerzo se orientará a conocer su cometido, funcionamiento y alcance. Con tal intención se estudiará un número importante de variables relacionadas con el papel que juegan los ciudadanos.

Una vez identificado el modelo participativo del PNBC, se buscará la mayor semejanza posible con alguno de los cuatro subtipos de participación general que se pro- 
ponen. Estos últimos se inspiran en algunos trabajos de la IUCN sobre gobernanza en espacios protegidos (Borrini-Feyerabend et al., 2013) y sobre participación pública del sistema de áreas marinas protegidas de Estados Unidos (Leigh, 2004). Y es que los autores citados dibujan una especie de línea continua de la gobernanza donde las instituciones gubernamentales ocupan un extremo y los actores sociales el extremo opuesto. El resultado surge de aplicar diferente protagonismo a la administración pública con respecto al papel que juegan los actores sociales en la gestión. Pero lo más interesante de estas aportaciones es su carácter progresivo que, en forma de gradientes, escenifica distintas manifestaciones de la gobernanza en los espacios protegidos.

Partiendo de las aportaciones antes citadas, y de otras más específicas para el PNBC (Díaz, 2013; Camilleri, Pérez-Hurtado y Gabbianelli, 2014) hemos avanzado de dos maneras. Por un lado, aumentando de forma notable el número de variables utilizadas para definir los subtipos participativos. Por otro, precisando y profundizando más en aquellas de las que se puede obtener información objetiva para los espacios naturales de Andalucía.

\section{El marco territorial y el marco legal específico para la participación ciudadana en el Parque Natural Bahía de Cádiz}

En primer lugar conviene describir, de forma sucinta al menos, el marco territorial del PNBC. Varias razones aconsejan tal proceder como se verá en páginas sucesivas. La más importante es la singularidad de su emplazamiento. En efecto, hay muy pocos parques naturales que se sitúen en el centro de una aglomeración urbana que, como esta, supera los 430.000 habitantes (IECA, 2015) repartidos entre cinco municipios, todos de cierta entidad poblacional: Cádiz, San Fernando, El Puerto de Santa María, Chiclana de la Frontera y Puerto Real. Así se explica que el espacio protegido de la Bahía de Cádiz esté literalmente rodeado de áreas residenciales de alta densidad, grandes industrias, infraestructuras de interés nacional y equipamientos metropolitanos. El segundo aspecto destacado es que la mitad de las 10.000 ha que tiene el parque son salinas mareales (la otra mitad está constituida por marismas y caños). Es decir, son espacios muy transformados por el ser humano desde hace siglos.

Por dichos motivos la autoridad político administrativa regional de los años ochenta de la anterior centuria vinculó su protección sólo al planeamiento urbanístico y territorial de la Bahía de Cádiz; en concreto lo encajó en el esquema de espacios libres (Zoido, 1982; Consejería de Obras Públicas y Transportes, 1986; Fourneau y Garrido, 1987; Chica, 2000). Incluso cabe recordar que, en los primeros borradores del entonces denominado "Inventario de Espacios Naturales de Andalucía", con el que la Comunidad Autónoma empieza a perfilar su futura red de espacios protegidos, no aparecía 
la Bahía de Cádiz. Para los representantes institucionales de la época, este espacio no reunía los suficientes atributos para hacerlo merecedor de protección con categoría de Parque Natural.

Por otro lado, algunos colectivos o asociaciones ecologistas, "Araucaria" de Cádiz, "Ortiga" de San Fernando y "Guadalete" de El Puerto de Santa María, expresaron desde 1984 una opinión contraria (Colectivo Ecologista Ortiga, Asociación Ecologista Guadalete y Colectivo Ecologista Pacifista Araucaria, 1984; Clavero, 1989; Merino, 1993). Incluso se movilizan para que las marismas y salinas de la Bahía de Cádiz fueran declaradas espacio natural protegido. Su iniciativa tuvo éxito. En una sesión plenaria del Parlamento de Andalucía en junio de 1989, la Bahía de Cádiz fue incluida en el Inventario de los Espacios Naturales de Andalucía ${ }^{1}$. La primera idea que trasmite este apunte histórico es que el esquema de espacios protegidos actual se explica, en gran medida, gracias a la inquietud social y al esfuerzo de algunos grupos organizados.

Lo anterior sucede en un ámbito urbano, donde la oferta de ocio es más generosa que en otro de naturaleza rural, y, por tanto, los ciudadanos tienen la opción de elegir otras alternativas recreativas. Se aduce este argumento porque es el que se utiliza para expresar el escaso conocimiento y apego que la población de la Bahía de Cádiz tiene por su espacio protegido. De todas formas, la distancia que existe entre ciudadanos y parque también se puede explicar porque, por un lado, las salinas han sido históricamente propiedades privadas y cerradas, y, en todo caso, con accesos públicos muy limitados. Pero, por otro, y esto tampoco conviene olvidarlo, las marismas fangosas tienen un carácter inhóspito que no invita, precisamente, al paseo y al relax. De ahí que las pocas áreas forestales de este parque, y los senderos ofertados en los últimos años, constituyan lugares muy concurridos por los ciudadanos.

$\mathrm{Al}$ anterior argumento cabe añadir otro: la crisis productiva que afecta al parque, derivada de las dificultades de las empresas acuícolas y salineras, ha casi vaciado de trabajadores y actividad las 5.000 ha de salinas. Las consecuencias se observan cuando se conoce un dato muy elocuente que procede del estudio sobre el estado de las salinas que hizo la dirección del parque en 2015: 48 de las 129 salinas censadas tienen importantes roturas en las "vueltas de afuera" o muros de protección mareal, impidiendo desarrollar su actividad con normalidad. Es posible que todas las causas descritas juntas hayan contribuido a una pérdida de interés social por el espacio protegido.

Las diferentes dimensiones del contexto a las que se ha hecho alusión (geográficas, territoriales, políticas, sociales y económicas) tienen gran trascendencia. Permiten en-

1. Ley 2/1989, de 18 de julio, por la que se aprueba el inventario de espacios naturales protegidos de Andalucía y se establecen medidas adicionales para su protección (Boletín Oficial de la Junta de Andalucía 60 de 27 de julio de 1989 y BOE 201 de 23 de agosto de 1989). 
cuadrar de forma más objetiva, en el espacio y en el tiempo, la relación de apego o desafecto de los ciudadanos con respecto al espacio protegido. En modo alguno prepara argumentos favorables a un cierto determinismo geográfico. Más bien al contrario ya que se asume la hipótesis de que existe un bajo nivel de cultura participativa. Y este es un asunto de primer orden. Entre otras razones porque el papel que juegan las instituciones públicas, para facilitar y acelerar el progreso desde una democracia formal y pasiva a otra con tintes más participativos y de movilización social, es una cuestión de responsabilidad política.

El Decreto 239/1997 regula en la actualidad la participación ciudadana en todos los parques naturales de Andalucía ${ }^{2}$. En dicho texto legal se determina la constitución, composición y funciones de las Juntas Rectoras, ya que estos son los órganos colegiados definidos para tal cometido. En concreto, "tendrán funciones de control, vigilancia y participación ciudadana y, asimismo, velarán por el cumplimiento de la normativa reguladora del Parque Natural, propondrán normas para una eficaz defensa de sus valores ecológicos, promoverán el desarrollo sostenible tanto en el interior como en el entorno del Parque Natural y realizarán cuantas gestiones estimen positivas y necesarias para el espacio natural".

Lo que sucede es que cuando se mencionan las funciones específicas, el control y vigilancia al que se refería antes el citado decreto, se expresan con una redacción muy cautelosa con respecto al papel de los ciudadanos: "informar (el Plan de Ordenación de Recursos Naturales, PORN), promover (el conocimiento y difusión de los valores del espacio protegido), emitir informe (sobre planes que afecten a los recursos naturales del parque), requerir información (de las actuaciones y actividades), proponer (ampliaciones de los límites), informar (el programa anual de actuaciones), someter al Consejero de Medio Ambiente (la propuesta de gastos de funcionamiento de la JR...)". Si exceptuamos algún apartado que hace referencia a la aprobación del Reglamento de Régimen Interior de la JR, solo existe otro que transfiera a la JR capacidades realmente decisorias; en concreto la aprobación del Plan Rector de Uso y Gestión.

El texto legal también describe la composición de la Junta Rectora de todos los parques naturales de Andalucía. Siguiendo un patrón bastante común a todos ellos, al de la Bahía de Cádiz le asigna 45 miembros, incluido el Presidente (que, por tradición, suele pertenecer a la Universidad de Cádiz). La distribución es la siguiente: 22 representantes de las administraciones públicas (Administración General del Estado, AGE, Junta de Andalucía, y Entidades Locales), 17 para representantes sociales (grupos conservacionistas, partidos políticos, asociaciones de diverso tipo) y 6 para representantes de los intereses empresariales. A su vez, la administración presenta el siguiente re-

\footnotetext{
2. Decreto 239/1997, de 15 de octubre, por el que se regula la constitución, composición y funciones de las Juntas Rectoras de los parques naturales (Boletín Oficial de la Junta de Andalucía 133/1997, de 15 de noviembre).
} 
parto: 10 representantes de la Junta de Andalucía, 5 de la AGE (interpretando que tanto el Consejo Superior de Investigaciones Científicas y la Universidad de Cádiz pertenecen, junto con los funcionarios de Defensa, y la Demarcación de Costas, a la AGE), y 6 de la Administración Local (5 municipios más la Diputación Provincial).

La segunda referencia legal para conocer el funcionamiento de la JR es la Resolución de la Dirección General de la RENPA (Red de Espacios Naturales Protegidos de Andalucía), por la que se publica el Reglamento de Régimen Interno de la JR del Parque Natural de la Bahía de Cádiz ${ }^{3}$. Las atribuciones señaladas para este órgano colegiado se detallan bastante bien aunque siguen la misma filosofía del Decreto 239. Prueba de ello es que la conjugación verbal de dichas funciones comienzan de forma similar: informar, colaborar, promover, facilitar, emitir informe, conocer, proponer, etc. Otros dos aspectos pueden destacarse de su contenido para el presente trabajo. Uno de ellos, artículo 13.1, es el que marca la periodicidad de las reuniones: "el pleno se reunirá en sesión ordinaria como mínimo dos veces al año, pudiendo convocarse otras con carácter extraordinario". El otro, artículo 28, señala las seis comisiones de trabajo en las que puede funcionar la JR: de coordinación administrativa, de infraestructura y servicios, de educación y cultura, de socioeconomía y turismo, de conservación, y de investigación.

\section{Hipótesis de trabajo, método y fuentes de información}

La hipótesis de partida de esta investigación radica en que no existe un modelo participativo para la gestión de los espacios protegidos de Andalucía, al menos claramente definido en favor de los ciudadanos. Esto último implica que los agentes sociales no gubernamentales son ajenos a la autoridad, al control real y a las decisiones importantes que rigen los destinos del espacio protegido. Se asume la afirmación de Sanz y Torres (2006) cuando subrayan que el funcionamiento de los espacios protegidos está alejado de la participación real y, por lo tanto, no es efectiva. Lo anterior no quiere decir que un parque deba ser gestionado, exclusivamente, por los agentes sociales. Pero entre esta opción extrema, dudosamente acertada, y la que entrega casi todo el poder de decisión a las instituciones gubernamentales, existen matices de cierta trascendencia. Y son estos matices los que diferencian a modelos democráticos meramente formales, de otros donde la participación pública forma parte de la cultura de gobernanza.

Por otro lado, la identificación del perfil participativo de un espacio protegido puede ayudarse de los diferentes gradientes de gestión que presenta el modelo general. Así, desde

3. Resolución de 15 de diciembre de 2000, de la Dirección General de la Red de Espacios Naturales Protegidos y Servicios Ambientales, por la que se dispone la publicación del Reglamento de Régimen Interior de la Junta Rectora del Parque Natural Bahía de Cádiz (Boletín Oficial de la Junta de Andalucía, 18 de enero de 2001). 
un extremo, donde las instituciones gubernamentales cobran protagonismo exclusivo, hasta el opuesto, donde los agentes de la sociedad local ostentan el control y la autoridad, se dibuja un continuo de gobernanza que permite observar distintos escenarios. Esto ya ha sido expresado por Borrini et al. (2014: 44 y 45) cuando, en su ensayo para la IUCN, refleja la relación que tienen los cuatro subtipos de gobernanza: gubernamental, compartida, privada y de comunidades locales. También Leigh (2004: 11), siguiendo trabajos anteriores de la Oficina para la Gestión de los Recursos Costeros y Oceánicos de Estado Unidos (perteneciente a Administración Nacional Oceánica y Atmosférica, NOAA por sus siglas en inglés), que tiene a su cargo el Programa de Áreas Marinas Protegidas, distingue cuatro niveles en el marco de un continuo de gobernanza que oscila desde la autoridad total de las agencias gubernamentales hasta el control por parte de los agentes sociales.

El método utilizado para comprobar las hipótesis de trabajo consiste en profundizar en el análisis y funcionamiento de la Junta Rectora del PNBC. El siguiente paso del método consiste en buscar la mayor similitud posible entre las características y funcionamiento de la JR del PNBC, y alguno de los modelos generales de participación que se han definido con tal propósito. Claro que es posible estudiar otras fórmulas de participación pública: voluntariado, campañas o actividades organizadas desde el parque, etc. Pero no cabe duda de que la mencionada institución constituye, por su carácter reglado y naturaleza representativa, el canal más importante que tienen los ciudadanos para ejercer su derecho a la participación en los asuntos del parque.

La principal fuente de información utilizada en trabajos de investigación sobre participación en espacios protegidos ha sido la entrevista. Las tesis doctorales de Torres (2003) para la Sierra de Baza y la de Bejarano (2011) para Doñana, son buenas muestras de ello. También la encuesta es muy socorrida cuando se trata de conocer la percepción de los diferentes agentes institucionales y sociales sobre algunos aspectos clave de la gobernanza como es la participación pública. Y el estudio que realizan Romagosa, Eagles y Buteau (2012) en algunos parques canadienses es muy ilustrativo al respecto.

En el caso de la Bahía de Cádiz se ha acudido a dos: a un cuestionario elaborado de forma exclusiva para la ocasión, y la atenta lectura de diferentes documentos públicos de origen institucional (actas de las Juntas Rectoras fundamentalmente, normativa existente, etc.). En las tablas 1 a 4 aparecen los resultados recogidos en las columnas de la derecha, con el encabezamiento denominado Actas y Cuestionario. Sobre el cuestionario cabe señalar que fue dividido en cuatro partes bien diferenciadas, tratando los siguientes temas a lo largo de 48 preguntas:

1. El órgano colegiado y su contexto (11 preguntas);

2. Quién participa (13 preguntas);

3. Sobre qué se participa (11 preguntas);

4. Cómo se participa (13 preguntas). 
Dicho cuestionario fue remitido por correo solo a 14 miembros de la actual JR. La razón de tal proceder se explica en que estos eran, en opinión del director conservador, los que mejor conocían el funcionamiento y trayectoria de la Junta Rectora, eran asistentes habituales a los plenos, solían proponer o involucrarse en determinadas iniciativas, etc. Pero, sobre todo, estos 14 miembros representaban muy bien a la mayoría de las instituciones públicas y privadas presentes en la JR. Desgraciadamente, las respuestas fueron insuficientes.

En un segundo intento, se optó por preguntar a miembros muy cualificados. Por un lado, se seleccionaron a los 4 directores conservadores del PNBC que ocuparon el cargo entre 1990 y 2015 (se descartó un quinto cuyo nombramiento estuvo vigente muy poco tiempo), que sí respondieron. Ellos conocen muy bien cómo se ha desarrollado la participación pública en la JR. Son los más relacionados y cercanos a los representantes sociales e institucionales debido a sus responsabilidades. Por esta razón sus opiniones son muy tenidas en cuenta.

El cuestionario también fue remitido y cumplimentado por dos de los miembros más antiguos y presentes en numerosas reuniones de las Juntas Rectoras: un representante empresarial y otro de la Demarcación de Costas. Se pretendía discriminar positivamente a aquellos participantes más presentes y activos en la JR a lo largo de los últimos quince años, condición que cumplen muy pocas personas. En este punto es preciso preguntarse si es posible que haya muchos miembros de la JR que puedan tener un profundo conocimiento de la misma. Varios argumentos obligan a esta reflexión: la frecuencia de las sesiones (una o dos al año), la relativa asistencia (menos del 50\% de media en los últimos 15 años), la rotación en el cargo de representación (4 años de vigencia). En cualquier caso, la intención final al interpretar los datos de las respuestas del cuestionario es obtener una orientación de tipo cualitativo, no llevar a cabo razonamientos precisos basados en resultados cuantitativos.

El valor de las respuestas a cada pregunta oscila entre 1 y 4. La puntuación más baja se identifica como la más cercana a una situación donde las instituciones públicas controlan completamente la gestión del parque. La nota más alta implicaría un modelo de participación en el que los ciudadanos son absolutos protagonistas de las decisiones y los designios del espacio protegido.

Para el análisis realizado de las actas, nuestra segunda fuente de información, se establecieron dos períodos temporales claramente diferenciados. El primero iría desde la creación del sistema de áreas protegidas, en 1989, hasta 1998. Y el segundo desde 1999 hasta 2015. Los hitos cronológicos se establecen a partir de la publicación del anteriormente comentado Decreto 239/1997. Éste entra en vigor a finales de enero de $1998^{4}$.

4. Corrección Boletín Oficial de la Junta de Andalucía 11/1998, de 29 de enero. 
Ello implica que hasta bien entrada esta fecha no fueron aplicadas las nuevas normas que establecían el funcionamiento y selección de los representantes sociales e institucionales en las Juntas Rectoras 5 .

La etapa inicial, de una década aproximadamente, tuvo la función de ensayar el modelo participativo creado. La siguiente recibiría, en forma de lecciones aprendidas, aquellas enseñanzas derivadas de la experiencia. Resulta obvio que el segundo período, 1999-2015, es el que más interesa a nuestra investigación. En este periodo se dispone de 26 actas de las diferentes reuniones de la Junta Rectora para su análisis.

La explotación de las dos fuentes, cuestionario y documentos, se hará al unísono. La razón es sencilla: aunque todas las preguntas fueron respondidas por los agentes sociales e institucionales, casi la mitad del cuestionario puede ser analizado, también, a partir del contenido de las actas, normas y otros documentos. Y de esta segunda fuente, no cabe duda, pueden obtenerse valiosos comentarios. Por otro lado, la percepción que pueden tener los miembros de la JR que han respondido el cuestionario, no siempre tiene porqué coincidir con la descripción de los hechos que refleja un acta.

\section{Resultados obtenidos}

\subsection{El órgano colegiado y su contexto}

La intención de este apartado es conocer algunas de las características de la JR y el contexto en el que se desenvuelve. En términos generales puede afirmarse que hay bastante coincidencia, aunque no plena, entre las respuestas obtenidas a partir de los cuestionarios y la procedente de fuentes documentales. La tabla 1 muestra cierto acuerdo en que su labor se desarrolla en un contexto con más cultura de gestión institucional que de participación ciudadana, y que está muy débilmente integrada en la sociedad de la Bahía de Cádiz.

El escaso conocimiento social puede ayudar a explicar la reducida presencia de representantes políticos e institucionales de primer nivel provincial. Este podría constituir un buen indicador sobre la relevancia política de la JR, y sobre si tiene prestigio e influencia en la vida pública. Las actas reflejan, por ejemplo, que el número de altos cargos que asisten, tanto de instituciones públicas como privadas, suele ser reducido. El resultado más repetido es 2-3. El Delegado provincial de la Consejería de Medio Ambiente y el representante del Ministerio de Defensa, son los altos cargos provinciales más asiduos.

5. Hasta entonces estuvieron vigentes las aprobadas en 1990, que fueron modificadas en 1995. 
Tabla 1. La Junta Rectora del PNBC y el contexto general de la participación pública

\begin{tabular}{|c|c|c|c|}
\hline & \multirow[b]{2}{*}{ Actas/varias } & \multicolumn{2}{|c|}{ Cuestionario } \\
\hline & & Moda & Media \\
\hline $\begin{array}{l}\text { 1. Cultura participativa. ¿Se desarrolla la Junta Rectora } \\
\text { en un contexto social con suficiente cultura } \\
\text { participativa? }\end{array}$ & - & 1 & 1,6 \\
\hline $\begin{array}{l}\text { 2. Base normativa. ¿Está suficientemente recogido el } \\
\text { papel y el funcionamiento de la Junta Rectora en } \\
\text { la legislación? }\end{array}$ & 3 & 3 & 2,6 \\
\hline $\begin{array}{l}\text { 3. Perfil funcional. ¿Se trata de un órgano colegiado } \\
\text { institucional y ejecutivo o participativo y consultivo? }\end{array}$ & 1 & 2 & 1,8 \\
\hline $\begin{array}{l}\text { 4. Integración social. ¿Conoce la sociedad local } \\
\text { suficientemente la Junta Rectora y su papel? }\end{array}$ & - & 1 & 1,1 \\
\hline $\begin{array}{l}\text { 5. Reconocimiento para los gestores. ¿Se considera } \\
\text { la Junta Rectora un órgano útil en la gestión del } \\
\text { parque por parte de los técnicos? }\end{array}$ & - & $2-3$ & 2,5 \\
\hline $\begin{array}{l}\text { 6. Consideración política. ¿Es considerado un órgano } \\
\text { de prestigio e influyente? }\end{array}$ & 1 & 2 & 2 \\
\hline $\begin{array}{l}\text { 7. Carácter integrado. ¿La Junta Rectora sirve para } \\
\text { interactuar y coordinarse con otras instituciones } \\
\text { públicas o privadas? }\end{array}$ & 2 & 3 & 2,1 \\
\hline $\begin{array}{l}\text { 8. Integración institucional. ¿Ocupa un lugar relevante } \\
\text { dentro del esquema general institucional? }\end{array}$ & 3 & $2-3$ & 2,5 \\
\hline $\begin{array}{l}\text { 9. Grado de autonomía. ¿Tiene la Junta Rectora } \\
\text { autonomía funcional o necesita de las instituciones } \\
\text { para funcionar? }\end{array}$ & 2 & 1 & 1,5 \\
\hline $\begin{array}{l}\text { 10. Recursos humanos. ¿Hay personal suficiente para } \\
\text { auxiliar las labores de la Junta Rectora? }\end{array}$ & - & 3 & 2,3 \\
\hline $\begin{array}{l}\text { 11. Medios económicos. ¿Existe un presupuesto adecuado } \\
\text { para el funcionamiento de la Junta Rectora? }\end{array}$ & 1 & 1 & 1 \\
\hline
\end{tabular}

La valoración oscila entre 1 (gestión institucional en exclusiva) y 4 (control ciudadano de la gestión).

Como sus reglas de funcionamiento están bien difundidas no extraña que sea percibido como un órgano colegiado de información y consulta. Es posible que esta sea la razón por la que es reconocida entre funcionarios y empleados públicos. En última instancia les facilita tomar el pulso del contexto social e institucional a la hora de tomar sus propias decisiones. 
Lo anterior guarda estrecha vinculación con el papel de la JR como instrumento de relación con otras instituciones, públicas y privadas. En este sentido las respuestas a los cuestionarios ofrecen una imagen más positiva de lo que las actas muestran: solo la mitad de las JR celebradas desde 1990 (13), reflejan la organización de reuniones previas (sea en grupos de trabajo, sea en comisión) o proponen reuniones futuras. Sobre la percepción de la calidad de estas comisiones conviene observar las respuestas a la pregunta 47 del cuestionario (Tabla 4). Ambas fuentes apuntan a que se trata de un órgano colegiado que ocupa un lugar relevante en el esquema de la Consejería correspondiente.

Si la mayor parte de las anteriores observaciones orientan acerca de una JR más cercana a la realidad institucional que a los ciudadanos, las respuestas referidas al grado de autonomía funcional, refuerzan, aún más, esta idea. Los medios económicos disponibles abundan en la misma conclusión de dependencia. A la pregunta sobre si ¿Existe un presupuesto adecuado para el funcionamiento de la JR? la respuesta es tan unánime y negativa como significativa. Sin embargo las actas reflejan una escasa preocupación por este tema (solo una vez fue tratado el tema con discrepancias). Ello podría orientar hacia un relativo nivel de conciencia participativa de los representantes ciudadanos, hacia un control institucional más que considerable, o a las dos razones al mismo tiempo. La opción más común, y menos imaginativa por cierto, fue la de asignar el escaso presupuesto destinado al funcionamiento de la JR a partidas relacionadas con el voluntariado y la educación ambiental (las cuales deberían contar con el suyo propio). Hay que señalar que desde hace algunos años la JR no dispone de presupuesto para gastos de funcionamiento como da a entender el Decreto 239/1997.

\section{2. ¿Quién participa?}

El presente apartado constituye una aproximación a los participantes. En este caso las actas de la JR suponen una fuente precisa de información para un buen número de preguntas (Tabla 2). En primer lugar hubo interés por saber la opinión sobre el número de representantes; y parece que hay cierto acuerdo entre los consultados acerca de que el número de personas que compone la JR es razonable y suficiente (hay que recordar que asciende a 45). Por lo que respecta al proceso de selección, los resultados del cuestionario indican que la mayoría de las respuestas valoran positivamente este proceso en favor de la legitimidad de la representación ciudadana. También el reconocimiento en la diversidad de los representantes facilita que los grupos sociales e institucionales importantes del área de influencia del parque puedan participar en la JR. Y es que estos aspectos parecen bien recogidos en la normativa comentada en apartados anteriores y aplicados en el PNBC. No obstante, convendría precisar algunos aspectos. Por ejemplo, incluir a las asociaciones de tipo cultural o definir mejor los criterios de representación de ciertos colectivos no incluidos en las grandes asociaciones (p. e. agrupaciones naturalistas locales que no pertenecen a Ecologistas en Acción, y que demandan su representación diferenciada de la gran coalición ecologista). 
Tabla 2. Quiénes participan en la Junta Rectora del PNBC

\begin{tabular}{|c|c|c|c|}
\hline & \multirow[b]{2}{*}{ Actas/varias } & \multicolumn{2}{|c|}{ Cuestionario } \\
\hline & & Moda & Media \\
\hline $\begin{array}{l}\text { 12. Número de representantes. ¿La composición de la } \\
\text { Junta Rectora tiene un número adecuado } \\
\text { de representantes? }\end{array}$ & 3 & 3 & 3,1 \\
\hline $\begin{array}{l}\text { 13. Proceso de selección. ¿El proceso de selección de } \\
\text { los representantes está bien definido } \\
\text { y asegura legitimidad? }\end{array}$ & 3 & 4 & 3 \\
\hline $\begin{array}{l}\text { 14. Diversidad de los representantes. ¿Están bien } \\
\text { representados todos los grupos sociales } \\
\text { e institucionales importantes? }\end{array}$ & 3 & 3 & 2,8 \\
\hline $\begin{array}{l}\text { 15. Equilibrio en la composición general. } \\
\text { ¿Está equilibradala representación entre } \\
\text { administración, sector empresarial y agentes sociales? }\end{array}$ & 1 & 2 & 1,8 \\
\hline $\begin{array}{l}\text { 16. Representación de las administraciones públicas. } \\
\text { ¿Está equilibrada la representación y asistencia } \\
\text { de las administraciones públicas? }\end{array}$ & 2 & $2-4$ & 2,6 \\
\hline $\begin{array}{l}\text { 17. Asistencia por grupos sociales. ¿Existe un equilibrio } \\
\text { razonable en la asistencia de los principales } \\
\text { grupos sociales? }\end{array}$ & 2 & $1-2$ & 2,1 \\
\hline $\begin{array}{l}\text { 18. Déficit de representación entre los asistentes. } \\
\text { ¿Se observa algún grupo social subrepresentado } \\
\text { O no representado? }\end{array}$ & 1 & $1-2-3$ & 2 \\
\hline $\begin{array}{l}\text { 19. Representantes clave. ¿Están los actores más } \\
\text { relevantes para el Parque Natural Bahía } \\
\text { de Cádiz representados? }\end{array}$ & - & 3 & 3,1 \\
\hline $\begin{array}{l}\text { 20. Asistencia general. ¿Puede considerarse el grado } \\
\text { de asistencia como aceptable? }\end{array}$ & 1 & $2-3$ & 2,5 \\
\hline $\begin{array}{l}\text { 21. Sesgos de la asistencia. ¿Existe algún sesgo en la } \\
\text { asistencia de los tres grandes grupos de representación? }\end{array}$ & 1 & 2 & 2 \\
\hline $\begin{array}{l}\text { 22. Nivel de interlocución. ¿Qué grado en la jerarquía } \\
\text { de sus instituciones suelen ostentar los asistentes } \\
\text { a las Junta Rectora? }\end{array}$ & 2 & 2 & 2,3 \\
\hline $\begin{array}{l}\text { 23. Grado de apertura. ¿Es abierto o cerrado el modelo } \\
\text { participativo del órgano colegiado? }\end{array}$ & 2 & 2 & 2,3 \\
\hline $\begin{array}{l}\text { 24. Grado de flexibilidad. ¿Se aplican los mecanismos } \\
\text { para agilizar la participación? }\end{array}$ & 3 & 2 & 2,1 \\
\hline
\end{tabular}

La valoración oscila entre 1 (gestión institucional en exclusiva) y 4 (control ciudadano de la gestión). 
En lo que respecta a los equilibrios de la composición de la JR, las opiniones fueron tan negativas como parecidas. Puede resultar una paradoja el hecho de que, en un órgano de participación ciudadana, sea tan evidente la excesiva presencia de la administración (22 representantes suponen casi la mitad del pleno). Incluso es posible que el sector empresarial esté poco presente. La percepción que tienen casi todos los directores conservadores y agentes sociales que respondieron al cuestionario es coincidente al detectar un desequilibrio favorable a las instituciones públicas.

Sobre estas hay sesgos, posiblemente lógicos, a favor de la representación de la administración regional. La práctica representativa seguida a través de las actas confirman que el peso de la Junta de Andalucía es, con diferencia, el mayor: es habitual que la suma de los representantes de la Junta de Andalucía sea igual o supere el correspondiente a las otras dos escalas administrativas juntas.

Si la cuestión es saber si existe un equilibrio razonable en la asistencia de los principales grupos sociales, las fuentes documentales no facilitan conclusiones claras. El volcado de datos de las actas se organizó estableciendo 3 grupos sociales bien diferenciados: ecologistas, partidos políticos y resto de asociaciones (deportivas, sindicales, consumidores, vecinos, etc.). Más bien cabe señalar cierta irregularidad en la asistencia de la mayoría. Como mucho, podría afirmarse que la asistencia de los representantes ecologistas es más patente a lo largo del período que la de otros colectivos con mayor representación (organizaciones sindicales y Federación Andaluza de Deportes, especialmente). De todas formas, como conjunto, los representantes sociales que han asistido a las JR desde 1999, han visto como el peso de su presencia se reducía desde el $28 \%$ en los cuatro primeros años, hasta el 25, 18 y 19\% en los siguientes cuatrienios.

Sobre los déficits de representación las actas no traslucen, por ejemplo, la participación de mariscadores y pescadores artesanales. Esta cuestión es importante habida cuenta de los servicios de abastecimiento y regulación que realizan los ecosistemas intermareales en relación a determinadas especies costero marinas (Chica y Barragán, 2011). Tampoco los intereses empresariales tienen una nutrida representación. Su presencia es escasa en los diferentes cuatrienios en lo que podría dividirse el período estudiado: 11, 9, 7, 11\% respecto al total de asistentes. Por otro lado, las mujeres suponen apenas una de cada cuatro asistentes. Y esto en el mejor de los casos; que coincide con los últimos años de la JR (en los anteriores la relación era más desfavorable aún). Resulta llamativo que estos déficits tan evidentes en las actas no se perciban de forma igualmente nítida en las respuestas a los cuestionarios.

También es curiosa la diferencia entre la percepción de aquellos que han respondido al cuestionario, y el resultado del estudio de las actas en relación a la asistencia general. Las actas reflejan que, de 45 componentes, a las reuniones de la JR han asistido algo menos de 22 personas de media en los últimos 15 años. El período de menor participación se registró entre 2010 y 2012 (con apenas 15-20 asistentes) y el mejor entre esta última fecha y 2014 (superando el 60\% de asistencia) (Figura 1). 


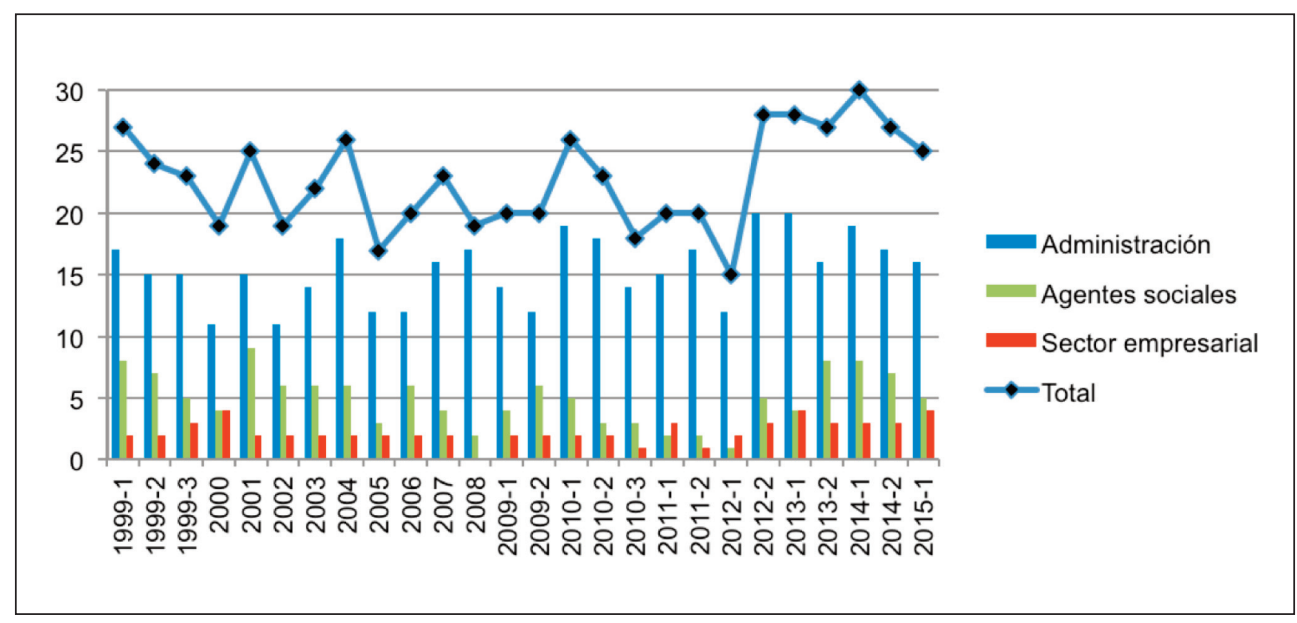

Figura 1. Evolución de la asistencia total y de cada grupo de actores a las reuniones de la Junta Rectora del Parque Natural Bahía de Cádiz (1999-2015).

Fuente: Elaboración propia.

No cabe duda de que una de las cuestiones más importantes para conocer el funcionamiento de las JR es la referida a los sesgos en la asistencia de los tres grandes grupos de representación. Las actas indican que durante todo el período analizado el 68,2\% de los asistentes pertenecían a la administración (con picos del 85 y 89\%), sobresaliendo, con diferencia, la Junta de Andalucía. En las últimas reuniones de la JR, el promedio de representación de la administración ronda el 70\%. Alguna reflexión puede hacerse a la luz de estos datos. Por ejemplo, se repite continuamente que las "opiniones" de las JR son casi siempre respetadas por parte de las instituciones públicas. La siguiente pregunta, retórica, nos parece muy pertinente ¿existe alguna diferencia entre las opiniones de la JR y las opiniones de las instituciones públicas con esta proporción de representantes que asisten a los plenos?

El nivel de interlocución es un aspecto clave para determinados acuerdos y decisiones. Con la intención de abordar este tema se preguntó sobre el grado que suelen ostentar los asistentes a las JR en la jerarquía de sus instituciones. Antes de cualquier comentario conviene traer a colación la pregunta 6 pues está muy relacionada con la presente cuestión. De las respuestas que ofrecían las actas se deduce que, desde el punto de vista político institucional, el número de asistentes de cierta categoría era muy bajo. Ahora, en cada acta se ha buscado el nivel de representación que ocupan los demás asistentes. La respuesta a esta pregunta ofrece pocas dudas: en 19 reuniones de la JR (de 26) se consideró que el nivel de representación en sus correspondientes instituciones u organizaciones era "bajo" ( ganización, representantes en organizaciones de reducido tamaño, profesores de uni- 
versidad o funcionarios del CSIC, jefes de servicio o de departamento en el caso de la administración) y en 7 "intermedio/bajo" (representantes de segundo nivel, vicerrectores, secretarios generales en departamentos de la administración, etc.).

Por último, hay dos preguntas que están relacionadas entre sí. Se refieren al grado de apertura de la JR y a su flexibilidad. En general, se identifica el modelo participativo de la JR con el de una institución con carácter representativo y relativamente cerrada. Lo anterior se compensa con cierto grado de flexibilidad. Entre otras razones porque el Reglamento de Régimen Interno de la JR del PNBC, señala, de forma explícita, entre las funciones del presidente, la de "requerir, en las reuniones de la Junta Rectora o Comisiones, la presencia, con voz pero sin voto, de aquellas personas que a su juicio puedan contribuir al mejor análisis de los temas incluidos en el orden del día”. Aunque existe otro procedimiento, el descrito suele ser el habitual para que personas ajenas a la JR puedan participar. Desde 1999 las actas de la JR del PNBC registran un total de 17 sesiones (de 26) en las que ha habido invitados. Otro dato de interés es que la aplicación de este mecanismo se hace más común a medida que pasa el tiempo.

\subsection{Sobre qué se participa}

En este apartado se analizan los asuntos tratados en la JR. La trascendencia y naturaleza de los mismos es algo que debe centrar la atención de cualquier análisis de la gestión de un espacio protegido. Hay dos observaciones sobre las que merece la pena realizar algún comentario. La primera es que casi todas las preguntas pueden obtener respuesta con la atenta lectura de las actas oficiales. Por otro lado, resulta conveniente subrayar la alta coincidencia en las respuestas ofrecidas por los directores conservadores del parque, pero también entre estos y los representantes del mundo empresarial y de la Demarcación de Costas.

En primer lugar nuestro interés se centró en la naturaleza de las iniciativas. Para eso había que estudiar en qué medida el orden del día reflejaba preocupaciones institucionales o sociales, o equilibrios entre unas y otras. La respuesta está muy condicionada por el hecho de que la JR se reúne dos veces al año, y son muy pocas las sesiones extraordinarias celebradas. Es decir, con escasas opciones de aplicar el criterio de urgencia o de oportunidad, y con una mayoría desbordante de asistentes procedentes del ámbito público, es lógico que las actas reflejen, sobre todo, inquietudes formales e institucionales, muy ligadas a procesos administrativos.

El papel de la participación ciudadana puede observarse también al tratar los temas objeto de debate. Para ello había que responder si en el orden del día aparecían asuntos relevantes para la gestión del PNBC (que puedan afectar a decisiones y tareas relacionadas con el control del parque, a inversiones, a cambios en las políticas públicas, etc.). Con estos criterios, y descontando aquellos puntos formales de cada sesión (pri- 
mero y último, aprobación de actas y ruegos y preguntas, respectivamente), el resultado de las actas es significativo. Cada uno de los cuatrienios en que fue dividido el período transcurrido desde 1999 indica que el porcentaje de temas relevantes tratados desciende a medida que pasa el tiempo: 36, 30, 32 y 23\%. Estos resultados contrastan con las respuestas a los cuestionarios (Tabla 3), que reflejan una opinión más favorable a considerar el funcionamiento de la JR acorde con las preocupaciones ciudadanas.

Una vez analizadas todas las actas puede hacerse una clasificación de los temas tratados, estableciéndose tres grupos acordes con su frecuencia de aparición:

a) Los más tratados, que coinciden con aquellos más relacionados con el funcionamiento y la misión del propio órgano de participación (16 sesiones).

b) Los tratados con una frecuencia intermedia, serían los económicos, 8 sesiones, y los estrictamente físico naturales y ambientales (9 sesiones)

c) Los temas menos tratados, se identifican con los culturales ( 5 sesiones), los sociales (3 sesiones), y los integrados o aquellos cuyo enfoque es cercano a la interpretación del parque natural como socioecosistema (2 sesiones).

Cuando se investigó el trabajo de la JR con respecto a los instrumentos de gestión del parque, las actas confirman que, ya sea en su versión más periódica (Memoria y Plan de Trabajo anual), o en un formato más estratégico (Plan de Ordenación de los Recursos Naturales, PORN; Plan Rector de Uso y Gestión, PRUG; Plan de Desarrollo Sostenible, PDS), se han tratado en prácticamente todas las sesiones de la JR. Este tipo de tareas no debe extrañar ya que dicha función es una de las principales que ostenta el órgano colegiado. No obstante, lo recogido en las correspondientes actas indica que, en casi todos los casos, el abordaje es puramente formal; no aparecen importantes debates o cambios sustanciales sobre lo que propone la institución pública.

Pero cuando el análisis busca conocer la opinión de la JR respecto a otros instrumentos de gestión que afectan al PNBC (planes de ordenación del territorio, planificación urbanística, planes de infraestructuras, etc.), las actas indican que solo en 12 ocasiones fueron examinados instrumentos como los señalados. Una opinión orientada hacia un escaso tratamiento de estos instrumentos también se puede encontrar en todas las respuestas de los directores conservadores del parque.

Por último cabe preguntar si, además de los objetivos de conservación y desarrollo, se pretenden reforzar los nexos de unión entre los representantes de la JR. Es decir, si se busca un modelo de participación social. Entendiendo éste como aquel que aspira a que las personas que habitan un espacio protegido desarrollen o refuercen vínculos entre ellas (cooperación, confianza...). Según algunos autores, así se podría conservar mejor dicho espacio al tiempo que se fortalece su cohesión como grupo social (Durán, 2005; Foronda, 2012), pero además mejorarían su eficiencia en el marco de un desarrollo más sostenible (Martínez y Romero, 2003). En las actas solo se observan 2 ocasiones en las que podría afirmarse que ha sucedido algo parecido. 
Tabla 3. Sobre qué se participa en la Junta Rectora del PNBC

\begin{tabular}{|c|c|c|c|}
\hline & \multirow[b]{2}{*}{ Actas/varias } & \multicolumn{2}{|c|}{ Cuestionario } \\
\hline & & Moda & Media \\
\hline $\begin{array}{l}\text { 25. Naturaleza de las iniciativas. ¿El orden del día refleja } \\
\text { preocupaciones institucionales o sociales? }\end{array}$ & 1 & 2 & 2,6 \\
\hline $\begin{array}{l}\text { 26. Trascendencia de los temas tratados. ¿Los temas } \\
\text { objeto de debate en el orden del día son relevantes } \\
\text { para la gestión del Parque Natural? }\end{array}$ & 2 & 3 & 3,3 \\
\hline $\begin{array}{l}\text { 27. Temas físico naturales y ambientales. ¿Se abordan } \\
\text { suficientemente temas relacionados con } \\
\text { el capital natural? }\end{array}$ & 2 & 3 & 2,8 \\
\hline $\begin{array}{l}\text { 28. Temas culturales. ¿Se abordan suficientemente } \\
\text { los temas relacionados con el capital cultural? }\end{array}$ & 1 & $2-3$ & 2,5 \\
\hline $\begin{array}{l}\text { 29. Temas de índole social. ¿Se debaten y hacen } \\
\text { propuestas suficientes relacionadas con } \\
\text { los aspectos sociales? }\end{array}$ & 1 & 2 & 2,1 \\
\hline $\begin{array}{l}\text { 30. Temas económicos. ¿Se tratan suficientemente } \\
\text { los asuntos vinculados a intereses empresariales? }\end{array}$ & 2 & 3 & 3 \\
\hline $\begin{array}{l}\text { 31. Temas de gobernanza. ¿Se tratan razonablemente } \\
\text { los asuntos relacionados con la misión y } \\
\text { el funcionamiento de la Junta Rectora? }\end{array}$ & 3 & 3 & 2,1 \\
\hline $\begin{array}{l}\text { 32. Temas integrados. ¿Se plantean temas donde } \\
\text { se interprete el Parque Natural Bahía de Cádiz } \\
\text { como un socioecosistema? }\end{array}$ & 1 & 3 & 2,3 \\
\hline $\begin{array}{l}\text { 33. Instrumentos de gestión propios. ¿Se analizan y } \\
\text { evalúan los principales instrumentos de gestión } \\
\text { del Parque Natural Bahía de Cádiz? }\end{array}$ & 2 & 2 & 2,3 \\
\hline $\begin{array}{l}\text { 34. Otros instrumentos de gestión de interés. } \\
\text { ¿Se analizan y evalúan otros instrumentos de gestión } \\
\text { que afectan al Parque Natural Bahía de Cádiz? }\end{array}$ & 2 & 2 & 2 \\
\hline $\begin{array}{l}\text { 35. Modelo de participación social. Además de otros } \\
\text { objetivos ¿se intenta reforzar los nexos de unión } \\
\text { entre representantes de la Junta Rectora? }\end{array}$ & 1 & 2 & 1,8 \\
\hline
\end{tabular}

La valoración oscila entre 1 (gestión institucional en exclusiva) y 4 (control ciudadano de la gestión). 


\section{4. ¿Cómo se participa?}

Este cuarto y último apartado se ocupa de analizar el modelo formal en el que se participa. En términos muy generales se interpreta como la búsqueda de algunos indicios de la calidad del proceso participativo. También aquí existe una gran coincidencia entre las opiniones de los cuatro directores conservadores y los representantes de las actividades empresariales y de la Demarcación de Costas.

Por el contrario no son bien considerados los aspectos relacionados con las facilidades disponibles que tienen los representantes de la JR para acceder a la información o para comunicarse entre ellos. Es decir, no existen canales propios y específicos para fomentar la participación (una página web, un foro en internet, etc.). No puede olvidarse, además, que se trata de herramientas elementales y con un muy bajo coste.

Sin perjuicio de un análisis pormenorizado se observa un contraste entre algunos valores altos y otros muy bajos para diferentes preguntas (Tabla 4). Por ejemplo, todas las fuentes valoran positivamente aspectos tales como la disponibilidad de la información que tienen los representantes de la JR (respecto a lo tratado en cada sesión), tiempo suficiente para el debate, la existencia de orden del día previo, calidad de esta misma información, etc. Hay acuerdo, por ejemplo, en reconocer que la calidad de la información que se suministra es la adecuada: suele estar actualizada, es sustancial, se entiende y sirve para que los representantes debatan.

Por último, se abordaron otros aspectos formales de la JR. Pero por formales no deben ser menos considerados; pues a veces son éstos los que explican la calidad de un proceso participativo. Por ejemplo, cuando se analizan los lugares donde se han desarrollado las sesiones, los resultados se resumen de la forma siguiente: 20 veces en San Fernando, 2 en Cádiz, 2 en El Puerto de Santa María, 1 en Chiclana y 1 en Puerto Real. Desde 2013 la JR se viene celebrando de forma rotatoria en cada municipio ribereño. Es lógico hasta cierto punto que la convocatoria favorezca a San Fernando, por estar allí situadas las oficinas del parque y, más recientemente, el Centro de Recepción de Visitantes. Pero es difícil explicar que los municipios con más superficie salinera y marismeña, Puerto Real y Chiclana (y, por tanto, con una percepción paisajística y territorial bien marcada), no tengan un mayor protagonismo en este sentido. Además, hay que recordar que estos municipios, a pesar de pertenecer a una aglomeración urbana, tienen todavía una identidad local muy definida.

También la frecuencia y el horario de los plenos son asuntos nada desdeñables. Es posible que, en general, los órganos colegiados que se reúnen frecuentemente, varias veces al año, por ejemplo, sean capaces de transmitir entre sus componentes importantes dosis de vinculación e interés. Hay que pensar que no es fácil mantener la atención por ciertos temas si, entre una reunión y otra de la JR, transcurren 6, 8, 10 o más meses. Las actas indican que, salvo la excepción de 1999 debida a los cambios nor- 
Tabla 4. Cómo se participa en la Junta Rectora del PNBC

\begin{tabular}{|c|c|c|c|}
\hline & \multirow[b]{2}{*}{ Actas/varias } & \multicolumn{2}{|c|}{ Cuestionario } \\
\hline & & Moda & Media \\
\hline $\begin{array}{l}\text { 36. Funcionamiento general. ¿La Junta Rectora se integra } \\
\text { en una red más amplia de participación? }\end{array}$ & 1 & 1 & 1,3 \\
\hline $\begin{array}{l}\text { 37. Calidad de la participación. ¿Tiene la Junta Rectora } \\
\text { a alguien especializado en procesos de gestión } \\
\text { participativa? }\end{array}$ & 1 & $1-2$ & 1,5 \\
\hline $\begin{array}{l}\text { 38. Accesibilidad general a la información. } \\
\text { ¿Puede acceder fácilmente cualquier ciudadano } \\
\text { a la información de la Junta Rectora? }\end{array}$ & 1 & 1 & 1,3 \\
\hline $\begin{array}{l}\text { 39. Disponibilidad de información. ¿Disponen los } \\
\text { representantes de la Junta Rectora de } \\
\text { suficiente información? }\end{array}$ & 3 & 3 & 2,8 \\
\hline $\begin{array}{l}\text { 40. Facilidades de acceso a la información. } \\
\text { ¿Disponen de canales propios los representantes } \\
\text { para un fácil acceso a la información? }\end{array}$ & 2 & 2 & 2,1 \\
\hline $\begin{array}{l}\text { 41. Facilidades para la comunicación. ¿Se dispone } \\
\text { de canales de comunicación propios para fomentar } \\
\text { la relación de la Junta Rectora? }\end{array}$ & 1 & $1-2$ & 1,5 \\
\hline $\begin{array}{l}\text { 42. Calidad de la información. ¿Se proporciona } \\
\text { información adecuada, en lenguaje comprensible } \\
\text { y con tiempo suficiente? }\end{array}$ & 3 & 3 & 3,1 \\
\hline $\begin{array}{l}\text { 43. Relación entre representantes y representados. } \\
\text { ¿Es fluida la relación entre representantes } \\
\text { y representados? }\end{array}$ & - & $1-2-3$ & 2 \\
\hline $\begin{array}{l}\text { 44. Modelo participativo. ¿Existe orden del día previo } \\
\text { y se facilita el debate? }\end{array}$ & 4 & $3-4$ & 3,5 \\
\hline $\begin{array}{l}\text { 45. Lugares. ¿La Junta Rectora se celebra en todos } \\
\text { los municipios de la Bahía de Cádiz? }\end{array}$ & 1 & 4 & 3,5 \\
\hline $\begin{array}{l}\text { 46. Frecuencia y horario. } \\
\text { ¿Se reúne a menudo la Junta Rectora? } \\
\text { ¿Se reúne en horario de mañana o tarde? }\end{array}$ & 1 & 2 & 3,5 \\
\hline $\begin{array}{l}\text { 47. Organización. ¿Se han organizado Comisiones } \\
\text { de trabajo y se muestran muy activas? }\end{array}$ & 1 & 2 & 2,5 \\
\hline $\begin{array}{l}\text { 48. Desarrollo de las sesiones. ¿Cuál es el tiempo de } \\
\text { duración de las reuniones? ¿Cuántos temas fueron } \\
\text { tratados? ¿Hubo votación? }\end{array}$ & 2 & 3 & 3,3 \\
\hline
\end{tabular}

La valoración oscila entre 1 (gestión institucional en exclusiva) y 4 (control ciudadano de la gestión). 
mativos ya comentados, la JR se ha reunido una vez al año hasta 2008, y a partir de ahí dos veces al año. En 20 ocasiones el horario elegido ha sido de tarde. Este aspecto tampoco debe ser despreciado en la participación ciudadana. Conviene recordar que las mañanas favorecen, claramente, a los representantes de la administración pública, ya que su asistencia coincidiría de forma plena con su horario laboral. Y para los representantes de instituciones privadas, sean de naturaleza social o empresarial, esta conveniencia no sería tan evidente.

La percepción del funcionamiento de las diferentes comisiones de trabajo no es muy positiva. La actividad de las mismas es valorada de forma relativamente baja por los directores conservadores. Lo cierto es que en las actas no hay muestras claras de una actividad importante.

En último lugar se analizó el desarrollo de las sesiones. Según las actas, estas oscilan entre 15 y 260 minutos, dependiendo de que sean extraordinarias (y haya un solo tema urgente) o de que haya varios puntos en el orden del día. Cada sesión dura, por término medio, poco más de dos horas, 134 minutos exactamente. La moda estadística es que el orden del día tenga bien 5 o bien 8 puntos para ser tratados. Los datos anteriores deben ser complementados con el hecho de que hay reuniones de la JR extraordinarias con 3 y otras ordinarias con hasta 9 puntos en el orden del día. Cada punto consume, por término medio, poco más de 26 minutos en su desarrollo. Bastantes de los asuntos debatidos que necesitan aprobación suelen conseguir el asentimiento (equivale a unanimidad), y no se procede a través de mano alzada o papeleta. Solo 6 veces, en los últimos quince años, ha tenido lugar una votación reflejada en las actas. Casi siempre estaban relacionadas con los candidatos a presidentes, o bien con la elección de los 3 representantes de reconocido prestigio o conocimiento del parque al que se refiere el artículo 3.2 del Decreto 239. Prácticamente nunca una propuesta hecha desde la administración pública fue rechazada o derrotada en una votación.

\section{Conclusiones y propuestas}

Una de las primeras conclusiones sobre la participación pública en los espacios protegidos se relaciona con las fuentes de información utilizadas para su estudio. En efecto, la explotación de las actas de las Juntas Rectoras ha demostrado ser de gran utilidad, por fiables, precisas y objetivas. Pero resulta paradójico que estas ventajosas características contrasten con su escasa utilización y aprovechamiento en investigaciones precedentes ${ }^{6}$.

\footnotetext{
6. El II Informe de Situación de la Red de Parques Nacionales (Organismo Autónoma de Parques Nacionales, 2012) incluye una evaluación de la gobernanza con técnicas de análisis de actas de los patronatos.
} 
Respecto a las conclusiones de contenido conviene reiterar algún matiz o mostrar cierta cautela. Es cierto que el estudio de un solo caso es incapaz de soportar conclusiones definitivas. No obstante, la homogeneidad institucional es una de las características dominantes en la RENPA, con escasas variantes en otros espacios naturales incluidos en la Red. En consecuencia, este ejercicio, aunque se centre en el PNBC, facilita la aproximación al perfil del modelo participativo en los espacios protegidos andaluces. La conveniencia de este trabajo viene reforzada, además, por la necesidad y urgencia de hacer un seguimiento preciso del funcionamiento y del papel de las Juntas Rectoras.

Buena parte de los resultados obtenidos en la presente investigación apuntan a una participación más aparente que real. Incluso es posible afirmar que el modelo vigente no es tanto de participación ciudadana como de encuentro institucional. A pesar de que la Junta Rectora, según el Decreto 239/1997, debe asumir "el control, vigilancia y participación ciudadana" en los espacios protegidos, en la práctica siguen prevaleciendo las funciones que señalaba el artículo primero del Decreto 11/1990, cuando establecía "funciones de coordinación de las administraciones públicas".

Se asiste a un modelo dirigido y tutelado por las instituciones públicas, muy alejado del control ciudadano. La composición de las Juntas Rectoras, con un esquema representativo muy sesgado en beneficio de las instituciones públicas, tiene mucho que ver con las anteriores afirmaciones. Los datos obtenidos señalan, además, un hecho comprobado: el funcionamiento cotidiano de estos órganos colegiados tiene efectos de amplificación del control institucional. También el grado de asistencia, la periodicidad, los temas tratados, la disponibilidad de canales de información, la accesibilidad a instrumentos de comunicación, etc. contribuyen a perfilar el modelo participativo del Parque Natural Bahía de Cádiz y, por extensión, de la Red de Espacios Naturales Protegidos de Andalucía (RENPA).

Las afirmaciones anteriores no contradicen prácticas impecablemente ejecutadas, pero solo desde un punto de vista formal, donde se incluye cierta flexibilidad en los procedimientos. Se trata de un modelo participativo dotado de soporte legal, pero diseñado para ejercer el control institucional. El modelo fue concebido, hace varios lustros, para que los ciudadanos estuvieran informados pero no fueran los responsables de las decisiones fundamentales que afectan al parque. Si lo anterior se encaja en un marco general donde la cultura participativa es débil, se comprende bien que haya perdurado sin reproche ni crítica social alguna.

Y lo sucedido no es solo responsabilidad de las autoridades político administrativas. Tampoco los representantes sociales y empresariales han tenido capacidad de organizarse, movilizarse y exigir un modelo más participativo. La prueba más elocuente es que el magro presupuesto disponible para la JR, cuando lo hubo, fuera destinado a labores de difusión de los valores del parque, pero no a la mejora del funcionamiento de una institución responsable de la participación ciudadana que no funcionaba. Y eso que im- 
presiona observar como los representantes sociales que han asistido a las JR desde 1999, reducen el peso de su presencia desde el $28 \%$ en los cuatro primeros años, hasta el 25 , 18 y $19 \%$ en los siguientes cuatrienios. ¿Desconocimiento, desinterés, falta de liderazgo social, o simplemente ausencia de cultura participativa en los asuntos públicos? En cualquier caso, en este marco la institución pública encuentra un escenario muy cómodo para mantener su control institucional. Lo paradójico es que, al mismo tiempo, puede afirmar que dispone de instrumentos al servicio del "control, vigilancia y participación ciudadana".

En la tabla 5 se han definido cuatro subtipos que podrían encajar con diferentes grados de participación ciudadana en la gestión de espacios protegidos. En un extremo se ha situado el modelo que hemos denominado "Dirigido", en el que las instituciones públicas absorben casi todo el protagonismo y responsabilidad en un parque. En el extremo opuesto está el "Autónomo", que sería aquel modelo que representa el poder ciudadano en su máxima expresión. Entre uno y otro aparece el "Tutelado" y el de "Transición", como exponentes de situaciones intermedias entre los dos primeros.

Sería acertado interpretar que se ha intentado plasmar una especie de orden de estado evolutivo entre el subtipo A y el D: desde una concepción más estatal o institucional de la gestión de un espacio protegido, a otra donde la participación ciudadana consigue el control (también, claro está, la responsabilidad). En términos generales corresponde aclarar que no es menos valorado y peor juzgado un modelo "Dirigido" que otro "Autónomo", ya que este aspecto dependerá del contexto social, económico y político. En todo caso orienta sobre las distintas posibilidades de participación ciudadana que existen.

Las variables utilizadas para este ejercicio aparecen en la primera columna. No coinciden de forma exacta con las analizadas en el punto anterior. Entre otras razones porque de las 48 preguntas del cuestionario, no todas sirven para determinar situaciones más o menos favorables a la participación ciudadana. Pero, en conjunto, cuando se cruzan respuestas procedentes de los 4 bloques estudiados a lo largo de este trabajo (órgano colegiado, quiénes, sobre qué y cómo se participa), los resultados obtenidos ayudan a observar donde se encaja el PNBC. En tal sentido, al menos 9 de las variables de la tabla 5 sugieren un modelo "Dirigido" mientras que 4 se orientan hacia otro "Tutelado". La conclusión general es, en cualquier caso, que estamos ante un modelo muy alejado de los dos subtipos de participación que sugieren control y responsabilidad ciudadana.

La última conclusión es la idea más sencilla y ha sido avalada por los resultados de la investigación: resulta imprescindible acometer, con cierta urgencia, mejoras de calado democrático en el modelo participativo de los espacios protegidos. Algunas cifras del PNBC no soportan una valoración objetiva: la media de asistencia durante los últimos 15 años no llega al 50\%, y eso representa menos de 22 personas; de ellas el 68\% pertenecía a la administración en sus diferentes escalas territoriales. La pregunta final, retórica claro, puede ser ¿es posible justificar la existencia de un órgano colegiado de participación ciudadana con solo 7 personas que se reúnen realmente una o dos veces al año? Porque este es el número medio de asistentes que los colectivos sociales y la administración han puesto a disposición de los plenos de la Junta Rectora. 





Las mejoras antes aludidas deben estar al servicio de un nuevo modelo de cogestión de espacios protegidos. No es viable un modelo en el que los ciudadanos y usuarios sigan alejados, o no sientan afecto por lo que hay que conservar. La gobernanza implica un nuevo rol de la administración en relación a los espacios de encuentro que les ofrece a los colectivos sociales y al sector empresarial. Por eso las Juntas Rectoras pueden avanzar en las mismas cuatro líneas que han sido estudiadas en páginas precedentes. No hace falta decir que todas ellas están interrelacionadas. Incluso es posible afirmar que sin el avance de unas el estancamiento de otras está casi asegurado:

1. Las Juntas Rectoras deben ser concebidas como órganos colegiados más autónomos, menos tutelados y dependientes de las instituciones públicas. Resulta obvio que deben centrarse en los asuntos de la gestión del parque. Pero también tienen que proyectar su papel hacia fuera, hacia una sociedad sobre la que tienen que ganar prestigio e influencia. Y eso es difícil conseguirlo sin estar dotadas de cierta autonomía, responsabilidad y medios, modestos pero mínimos. En realidad se hace referencia a una cuestión que trasciende del propio parque natural; se trata de nuestra cultura participativa, de la construcción de instituciones donde los ciudadanos sean corresponsables de los asuntos públicos, que son los suyos.

2. Los representantes en la Junta Rectora deben reflejar mejor el contexto territorial, social, económico y, por supuesto, político en el que se encuentra el espacio protegido. En el caso de los parques litorales, por ejemplo, existen usuarios de servicios ecosistémicos y administraciones públicas poco o nada representadas. Además, el exagerado desequilibrio entre miembros de la JR pertenecientes a la administración, por un lado, y colectivos sociales y sector empresarial, por otro, es preciso corregirlo. Esta propuesta no contradice la permanencia, incluso mejora, de un órgano colegiado de coordinación institucional; pero independiente de la Junta Rectora. También la escasa asistencia de los representantes de estos dos últimos vértices del triángulo de la gobernanza, exige medidas que dinamicen su presencia y actividad en los plenos. Y lo mismo sucede con grupos que no están suficientemente representados por una razón u otra (mujeres, mariscadores, pescadores artesanales, desempleados, etc. en el caso del PNBC). Es posible que, en caso de seguir como hasta la fecha, el absentismo ciudadano pueda restar sentido a las JR. Y la administración, especialmente la comunidad autónoma por sus competencias en los espacios protegidos, no puede ignorar esta situación.

3. Los temas objeto de debate en las JR tienen que ir cambiando de forma progresiva. Desde los más ligados a asuntos formales, que son los que predominan en los últimos años, hacia otros que reflejen mejor los problemas e inquietudes sociales: planes y proyectos sectoriales de naturaleza pública, iniciativas empresariales de alcance, accesos públicos, empleo, equidad en la distribución de los beneficios que genera el parque, decisiones sobre el uso público, etc. Lo anterior no impide que en el enfoque de los temas tratados prime una interpretación de espacio integrado y un modelo de participación social. De esta forma la mayor cohesión de los grupos existentes aumentará la eficacia de las iniciativas de conservación. 
4. En el cómo se participa se observan grandes carencias. Sobre algunas de ellas es posible avanzar con mayor dotación de medios o con nuevos mecanismos: funcionamiento de la Junta Rectora en red, trabajo de especialistas en dinámicas participativas (que podrían ser contratados, por ejemplo, con los presupuestos asignados a las JR), plataformas para el acceso a una información que mejore la eficiencia y transparencia de los procesos participativos, canales para la mejora de la comunicación entre representantes, y entre éstos y el público en general, etc. Otras deben acompañar a una mejora sustancial de la cultura participativa: protagonismo de las comisiones o grupos de trabajo, realización de propuestas bien definidas que merezcan debate y posterior votación, etc.

Las propuestas anteriores pueden ser articuladas por la Consejería de Medio Ambiente a través de una línea de trabajo específicamente dirigida a mejorar los estándares de gobernanza en los espacios naturales protegidos de la región.

\section{Bibliografía}

Abrams, P., Borrini-Feyeranbend, G., Gardner, J. y Heylings, P., 2003. Evaluating governance: A handbook to accompany a participatory process for a protected área. Ottawa, Parks Canada and TILCEPA, $120 \mathrm{p}$.

Becken, S. y Job, H., 2014. Protected Areas in an era of global-local change. Journal of Sustainable Tourism, vol. 22, no 4, p. 507-527.

Bejarano, J.F., 2011. La gestión compartida en los espacios naturales protegidos. Análisis sociológico de la participación ciudadana en Doñana. Tesis doctoral dirigida por Carmen Sanz López y Adolfo José Torres Rodríguez. Granada, Universidad de Granada, 591 p.

Borrini-Feyerabend, G., Dudley, N., Jaeger, T., Lassen, B., Pathak-Broome, N., Phillips, A. y Sandwith, T., 2015. Gobernanza de áreas protegidas: de la comprensión a la acción. Gland, Suiza, UICN (№ 20 Serie Directrices buenas prácticas en áreas protegidas), XVI + 123 pp.

Camilleri, S., Pérez-Hurtado, A. y Gabbianelli, G., 2014. Multiple DPSI frameworks for support of integrated research: a case study of the Bahía de Cádiz Nature Park (Spain), Journal of Coastal Conservation, Planning and Management, vol. 18, ํํ 5 .

Chica Ruiz, J.A., 2000. Los instrumentos de planificación física en relación con los espacios naturales protegidos en la Bahía de Cádiz. [CD-Rom]. Cádiz, Servicio de Publicaciones de la Universidad de Cádiz, 150 p.

Chica, J.A. y Barragán, J.M., 2011. Estado y tendencia de los servicios de los ecosistemas litorales de Andalucía. Sevilla, Junta de Andalucía, 112 p.

Clavero, J., 1989. El Parque Natural de la Bahía de Cádiz. En Federación Ecologista Pacifista Gaditana (ed.). Espacios naturales de la provincia de Cádiz. Cádiz, FEPG.

Colectivo Ecologista Ortiga, Asociación Ecologista Guadalete y Colectivo Ecologista Pacifista Araucaria, 1984. Parque Natural y Reservas Integrales de la Bahía de Cádiz. Cádiz (Resumen del Proyecto). 
Consejería de Obras Públicas y Transportes, 1986. Plan Especial de Protección del Medio Físico y Catálogo de Espacios y Bienes Protegidos de la provincia de Cádiz. Sevilla, Junta de Andalucía, $160 \mathrm{p}$.

Dearden, O., Bennett, M. y Johnston, J., 2005. Trends in global protected area governance, 19922002. Environmental Management, vol. 36, no 1, p. 89-100.

Díaz Garduño, B., 2013. Participación pública en el Parque Natural Bahía de Cádiz. Trabajo Final de Máster en Gestión Integrada de Áreas Litorales dirigido por Juan Manuel Barragán Muñoz. Cádiz, Universidad de Cádiz, 50 p.

Durán, L., 2005. Participación social y conservación, En Barahona, A. y Almeida, L. (Coord.). Educación para la conservación. México D.F., Facultad de Ciencias, Universidad Autónoma de México, p. 66-76.

Europarc-España, 2002. Plan de Acción para los espacios naturales protegidos del Estado Español. Madrid, Fundación Fernando González Bernáldez, 168 p.

Europarc-España, 2007. Enredando. Herramientas para la comunicación y la participación social en la gestión de la red Natura 2000. Madrid, Fundación González Bernáldez, 216 p.

Europarc-España, 2014. Esparc 2014. Actas del XVIII Congreso Europarc-España. Madrid, Fundación Fernando González Bernáldez, 135 p.

Foronda Robles, C. (Coord.), 2012. Capital social en espacios naturales protegidos. Pamplona, Ulzama Ediciones, 385 p.

Fourneau, F. y Garrido, J. (Dir.), 1987. Evolución de los paisajes y ordenación del territorio en Andalucía occidental. Bahía de Cádiz. Cádiz, Diputación Cádiz y Casa Velázquez, 237 p.

García, M.R. y Montes, C., 2010. AN + 20. El desafío de la gestión de los espacios naturales de Andalucía en el siglo XXI. Una Cuestión de Valores. Sevilla, Consejería de Medio Ambiente (Junta de Andalucía), 166 p.

IECA (Instituto de Estadística y Cartografía de Andalucía), 2015. Padrón Municipal de habitantes. Cifras oficiales de población municipal 2014. Sevilla, Consejería de Economía, Innovación, Ciencia y Empleo (Junta de Andalucía).

Jones, P.J., Qiu, W. y De Santo E.M., 2011. Governing Marine Protected Areas - Getting the Balance Right. Journal of Sustainable Tourism, vol. 22, no 4, p. 507-527.

Leigh Kessler, B., 2014. Stakeholder Participation: A Synthesis of Current Literature. Prepared by the National Marine Protected Areas Center in cooperation with the National Oceanic and Atmospheric Administration Coastal Services Center.

Martínez, J. y Romero, R., 2003. Repercusión de los espacios protegidos en la economía española. Serie Geográfica, no 11 , p. 41-60.

Merino, L., 1993. El Parque Natural de la Bahía de Cádiz: singularidad y pervivencia de un espacio protegido. Cuadernos de Geografía de la Universidad de Cádiz, no 4, p. 31-51.

Organismo Autónomo de Parques Nacionales, OAPN, 2012. Segundo Informe de situación de la Red de Parques Nacionales (2007-2010), Madrid, Ministerio de Agricultura, Alimentación y Medio Ambiente, 166 p. 
Pomeroy, R.S., Parks, J.E. y Watson, L.M., 2006. Cómo evaluar una AMP. Manual de Indicadores Naturales y Sociales para Evaluar la Efectividad de la Gestión de Áreas Marinas Protegidas. Gland y Cambridge, UICN, 216 p.

Romagosa, F., Eagles, F.J. y Buteau, W., 2012. Evaluación de la gobernanza en los espacios naturales protegidos. El caso de la Columbia británica y Ontario (Canadá). Anales de Geografía, vol. 32, no 1, p. 133-151.

Sanz, C. y Torres, A., 2006. Gobernabilidad en las áreas protegidas y participación ciudadana. Papers, Revista de Sociología, 82, p. 141-161.

Secretaría de la Convención de Ramsar, 2010. Aptitudes de participación: Establecimiento y fortalecimiento de la participación de las comunidades locales y de los pueblos indígenas en el manejo de los humedales. Manuales Ramsar para el uso racional de los humedales (4⿳⺈⿴囗十一 edición). Gland, Secretaría de la Convención de Ramsar vol. 5. 120 p.

Torres, A.J., 2003. Estudio sociológico de los espacios naturales protegidos: de la conservación a la sostenibilidad. Tesis doctoral dirigida por José Sánchez Alhama y Carmen Sanz López. Granada, Universidad de Granada, 477 p.

Zoido Naranjo, F., 1982. Bahía de Cádiz. Bases para la coordinación del planeamiento urbanístico. Cádiz, Diputación Provincial de Cádiz, 200 p. 
